\title{
A NOVEL APPROACH TO THE KPZ DYNAMICS*
}

\author{
H.S. WiO \\ Instituto de Física de Cantabria (UC and CSIC) \\ 39005 Santander, Cantabria, Spain \\ R.R. DEZA \\ Instituto de Física Mar del Plata (UNMdP and CONICET) \\ 7600 Mar del Plata, Argentina \\ J.A. REVELLI \\ Instituto de Física E. Gaviola (UNC and CONICET) \\ 5000 Córdoba, Argentina \\ C. Escudero
}

Departament of Mathematics, Universidad Autónoma de Madrid and ICMAT 28049 Madrid, Spain

(Received May 20, 2013)

We discuss a tentative path-integral approach to numerically follow the scaling properties of the mean rugosity (and other typical averages) of an interface whose growth is described by the Kardar-Parisi-Zhang equation. It resorts to functional minimization and a cellular automata-like algorithm, and can be regarded as a kind of importance-sampling approach. This method is intended to predict the crossover time as a function of the coefficient of the nonlinear term, through the comparison of the weight of the different terms in the "stochastic action".

DOI:10.5506/APhysPolB.44.889

PACS numbers: 05.40.-a, 05.45.-a, 68.35.Ct, 81.15.Aa

* Presented at the XXV Marian Smoluchowski Symposium on Statistical Physics, "Fluctuation Relations in Nonequilibrium Regime", Kraków, Poland, September 10-13, 2012. 


\section{Introduction}

One of the greatest accomplishments of statistical physics in the $20^{\text {th }}$ century has been the classification of equilibrium critical phenomena into universality classes depending solely on the dimensionality of the order parameter, and that of the embedding space. Regrettably, a similar achievement regarding nonequilibrium systems has been reluctant up to now.

The main tool for classifying equilibrium critical phenomena has been the renormalization group, based on the scaling behavior of the system's correlation functions under partial re-summations of the partition function implying scale changes, be in real or in momentum space [1]. Although the first implementation looks more intuitive, the second one - appealing to a field-theoretic description of the system - turned out to be more universal. With this background, it was highly desirable to dispose of a reliable field-theoretic description of a representative enough, nonequilibrium system. The stochastic, nonlinear partial differential equation, named after Kardar, Parisi and Zhang (KPZ) [2-4], has become the paradigm of such a (mesoscopic) description. In fact, it is known to represent a large and robust class of nonequilibrium critical phenomena.

From a theoretical point of view, the KPZ equation has many interesting properties, like its close relationship with the Burgers equation [5] or with a diffusion equation with multiplicative noise, whose field $\phi(x, t)$ can be interpreted as the restricted partition function of the directed polymer problem [6]. Most of the efforts put on investigating the behavior of its solutions concentrated in obtaining the critical exponents in one or more spatial dimensions [7-15]. Other questions of interest are the development of suitable algorithms for its numerical integration [13], the construction of particular solutions [16-18], unveiling the critical properties of this equation when set on growing domains [19, 20], etc.

Among the classical theoretical developments concerning this equation $[3,4]$, two have attracted our attention in recent works. One is the scaling relation $\alpha+z=2$, which is expected to be exact for the KPZ equation in any dimension. The exactness of this relation has been traditionally attributed to the Galilean invariance of the KPZ equation (related to the tilting of the interface). The conjectured central role of this symmetry has however been challenged in this as well as in different nonequilibrium models both from a theoretical [21-24] and a numerical [25-27] point of view. The other one is the generally accepted lack of existence of a suitable functional that allowed formulating the KPZ equation as a gradient flow. Regarding this last point, it was shown in [28] that the deterministic KPZ equation

$$
\partial_{t} h(x, t)=\nu \nabla^{2} h(x, t)+\frac{\lambda}{2}(\nabla h(x, t))^{2},
$$


where $\nu$ represents the surface tension and $\lambda$ the mean growth velocity, admits the Lyapunov functional

$$
\mathcal{F}[h]=\frac{\lambda^{2}}{8 \nu} \int \exp \left[\frac{\lambda}{\nu} h(x, t)\right]\{\nabla h(x, t)\}^{2} d x,
$$

such that the full KPZ equation, driven with effective intensity $\varepsilon$ by a Gaussian noise $\xi(x, t)$ of zero mean, unit variance, and white in space and time $\left[\left\langle\xi(x, t) \xi\left(x^{\prime}, t^{\prime}\right)\right\rangle=\delta\left(x-x^{\prime}\right) \delta\left(t-t^{\prime}\right)\right]$ can be written as

$$
\partial_{t} h(x, t)=-\Gamma[h] \frac{\delta \mathcal{F}[h]}{\delta h(x, t)}+\sqrt{\varepsilon} \xi(x, t)
$$

with $\Gamma[h]=\left(\frac{2 \nu}{\lambda}\right)^{2} e^{-\frac{\lambda}{\nu} h(x, t)}$. The Lyapunov property $\dot{\mathcal{F}}[h] \leq 0$ is straightforwardly checked, with $\dot{\mathcal{F}}[h]=0$ achieved by constant (in space) functions.

Another Lyapunov functional (which we call the nonequilibrium potential) can also be formally defined [28]

$$
\Phi[h]=\frac{\nu}{2} \int d x(\nabla h)^{2}-\frac{\lambda}{2} \int d x \int_{h_{\mathrm{ref}}}^{h(x, t)} d \psi(\nabla \psi)^{2},
$$

allowing to formally write the KPZ equation as a stochastically forced gradient flow

$$
\partial_{t} h(x, t)=-\frac{\delta \Phi[h]}{\delta h(x, t)}+\sqrt{\varepsilon} \xi(x, t) .
$$

The Lyapunov property $\dot{\Phi}[h]=-\left(\frac{\delta \Phi[h]}{\delta h(x, t)}\right)^{2} \leq 0$ follows from a formal computation. We have explored such a variational approach to the KPZ equation by means of a functional expansion of $\Phi[h]$, leading to an effective kinetic equation for the fluctuations which was in turn submitted to a dynamic renormalization group analysis [29]. This is in the spirit of the variational approach to the Sun-Guo-Grant [30] and the Villain-Lai-Das Sarma [31, 32] equations, developed in [33, 34] by means of a geometric construction, and put in more rigorous terms in [35, 36].

Here we discuss a different and new point of view. Motivated on the one hand by the knowledge of the KPZ's nonequilibrium potential [28], and on the other by the functional integral approach used in [37], we have done a tentative numerical analysis of the "stochastic action" (or Onsager-Machlup functional) that arises in a path integral approach to the KPZ propagator. We have mainly focused in identifying the different dynamical regimes, as well as in a possible characterization of the crossover time between the 
Edward-Wilkinson (EW) and the KPZ regimes. The method is thoroughly derived in Sec. 2, whereas the remaining two sections are devoted to the numerical results and the conclusions. We already remarked that this method is affected by a spurious instability that affects the long time results. We leave the search of possible solutions to this problem for the future.

\section{Theoretical foundation of the method}

For simplicity, we here consider only a 1D substratum and also disregard an eventual constant term $F$. This equation is, of course, nonlinear but our aim is not finding explicit solutions but rather, obtaining the scaling properties of some typical averages as e.g. the mean rugosity. These can be calculated with the aid of the solution of the associated Fokker-Planck equation (FPE, which indeed exists since the process, being driven by a white noise, is Markovian). This solution of a linear equation - the conditional probability density function (pdf) of the process, given an initial condition - is the object of which we seek a path-integral representation.

As it is known, any path-integral representation is defined as the limit of a discrete (matrix) representation. Now, there is a whole family (identified by some parameter $\alpha$ ) of discrete representations corresponding to the same continuous one. Although for the calculation we shall use particular values of $\alpha$, we must consider the general case in seeking the form of the Lagrangian [38, 39].

The starting point is to rewrite the KPZ equation in discrete form. Whereas time discretization is mandatory to make sense out of a path integral, space discretization is usually resorted to in numerical simulation. Subscripts $j, \mu$ indicate space- and time-coordinates, respectively. As discussed elsewhere [25-27], in the spatially discrete KPZ equation, one must resign either Galilean invariance or the Hopf-Cole mapping onto a diffusion equation (with multiplicative noise). In accordance with previous opinions [22, 23], our numerical study [25, 26] reveals that breaking Galilean invariance does not take the system out of the KPZ universality class; instead (in order not to resign the Hopf-Cole mapping, a cherished theoretical property of the KPZ equation), the discrete versions of the Laplacian and the squared gradient must be related in a precise fashion: if e.g. the Laplacian is written as $\left[\left(h_{j+1, \mu}-h_{j, \mu}\right)-\left(h_{j, \mu}-h_{j-1, \mu}\right)\right]$ (we take, for simplicity, the lattice spacing as 1$)$, then the squared gradient must be written as $\frac{1}{2}\left[\left(h_{j+1, \mu}-h_{j, \mu}\right)^{2}+\left(h_{j, \mu}-h_{j-1, \mu}\right)^{2}\right]$. Hence, we rewrite Eq. (1) as

$$
\dot{h}_{j}(t)=\boldsymbol{L}_{j}(\{h\})+\sqrt{\varepsilon} \xi_{j}(t),
$$


with

$$
\boldsymbol{L}_{j, \mu}=\nu\left(h_{j+1, \mu}-2 h_{j, \mu}+h_{j-1, \mu}\right)+\frac{\lambda}{4}\left[\left(h_{j+1, \mu}-h_{j, \mu}\right)^{2}+\left(h_{j, \mu}-h_{j-1, \mu}\right)^{2}\right] \text {. }
$$

As argued above, we should write the time discretization $t=M \tau$ in the following form

$$
h_{j, \mu+1}-h_{j, \mu} \approx \tau\left[\alpha \boldsymbol{L}_{j, \mu+1}+(1-\alpha) \boldsymbol{L}_{j, \mu}\right]+\sqrt{\varepsilon}\left(W_{j, \mu+1}-W_{j, \mu}\right),
$$

where $W_{j}$ is a Wiener process at site $j$. The last equation can be rewritten as

$$
W_{j, \mu}=\sqrt{\varepsilon}\left\{h_{j, \mu}-h_{j, \mu-1}-\tau\left[\alpha \boldsymbol{L}_{j, \mu}+(1-\alpha) \boldsymbol{L}_{j, \mu-1}\right]\right\}+W_{j, \mu-1} .
$$

Accordingly, the Jacobian of the transformation $\eta \rightarrow h$ (namely from noiseto height variable) is

$$
\begin{aligned}
\mathcal{J}=\operatorname{det}\left(\frac{\partial W_{j, \mu}}{\partial h_{k, \lambda}}\right) & =\Pi_{j, \mu}\left[\sqrt{\varepsilon}\left(1-\tau \alpha \frac{\partial \boldsymbol{L}_{j, \mu}}{\partial h_{j, \mu}}\right)\right] \\
& \approx \varepsilon^{-\frac{N M}{2}} \exp \left(-\tau \alpha \sum_{j, \mu} \frac{\partial \boldsymbol{L}_{j, \mu}}{\partial h_{j, \mu}}\right)
\end{aligned}
$$

where the last expression is an approximation valid for $\tau \rightarrow 0, N$ is the number of sites and $M$ the number of time steps. From Eq. (7), the derivative indicated in the last expression of Eq. (10) is

$$
\frac{\partial \boldsymbol{L}_{j, \mu}}{\partial h_{j, \mu}}=-2 \nu-\frac{\lambda}{2}\left(h_{j+1, \mu}-2 h_{j, \mu}+h_{j-1, \mu}\right)
$$

hence the exponent reads

$$
\begin{aligned}
-\tau \alpha \sum_{j, \mu} \frac{\partial \boldsymbol{L}_{j, \mu}}{\partial h_{j, \mu}} & =\tau \alpha \sum_{j, \mu}\left(2 \nu+\frac{\lambda}{2}\left[h_{j+1, \mu}-2 h_{j, \mu}+h_{j-1, \mu}\right]\right) \\
& =2 \nu \alpha N t+\tau \alpha \frac{\lambda}{2} \sum_{j, \mu}\left[h_{j+1, \mu}-2 h_{j, \mu}+h_{j-1, \mu}\right] .
\end{aligned}
$$

The contribution indicated in the last line must be included in the action. However, we already see that the second term is $\mathcal{O}(\tau)$ so for $\tau \rightarrow 0$ it may be negligible against more singular contributions.

Hereafter, we regard the interface configuration at time $\mu$,

$$
\{W\}=\left(W_{1, \mu}, W_{2, \mu}, \ldots, W_{j, \mu}, \ldots, W_{N, \mu}\right)
$$


as a "state". The transition pdf from an initial state $\left\{W_{0}\right\}$ to a final one $\left\{W_{\mathrm{f}}\right\}$ is $[38,39]$

$$
\begin{aligned}
P\left(\left\{W_{\mathrm{f}}\right\}, t \mid\left\{W_{0}\right\}, t_{0}\right)= & \int \mathcal{D}[W] \exp \left[-\frac{1}{\varepsilon} \int d t \int d x\left(\frac{\partial W_{x, s}}{\partial s}\right)^{2}\right] \\
= & \int \ldots \int\left(\frac{1}{2 \pi \varepsilon \tau}\right)^{N M / 2} d W_{1,1} d W_{1,2} \ldots d W_{j, \mu} \ldots d W_{N, M} \\
& \times \exp \left[-\frac{1}{2 \varepsilon \tau} \sum_{j, \mu}\left(W_{j, \mu+1}-W_{j, \mu}\right)^{2}\right] .
\end{aligned}
$$

We shall not work out explicitly here the derivation of $P\left(\left\{h_{\mathrm{f}}\right\}, t \mid\left\{h_{0}\right\}, t_{0}\right)$, the transition pdf between patterns $h_{0}(x)$ at time $t=t_{0}$ and $h_{\mathrm{f}}(x)$ at time $t=t_{\mathrm{f}}$. According to [38], it can be written as

$$
\begin{aligned}
& \int_{-\infty}^{\infty} \ldots \int_{-\infty}^{\infty}\left(\frac{1}{2 \pi \varepsilon \tau}\right)^{\frac{N M}{2}} d W_{1,1} d W_{1,2} \ldots d W_{j, \mu} \ldots d W_{N, M} \\
& \times \delta\left(\left\{h_{\mathrm{f}}\right\}-\left\{h_{N, M}\right\}\right) \exp \left[-\frac{1}{2 \varepsilon \tau} \sum_{j, \mu}\left(W_{j, \mu+1}-W_{j, \mu}\right)^{2}\right] .
\end{aligned}
$$

Using the Jacobian calculated in Eq. (10) and the fact that from Eq. (12)

$$
P\left(\left\{W_{\mathrm{f}}\right\}, t \mid\left\{W_{0}\right\}, t_{0}\right) \Delta\left\{W_{j, \mu}\right\}=\left(\frac{1}{2 \pi \varepsilon \tau}\right)^{\frac{N M}{2}} e^{-\frac{1}{2 \varepsilon \tau} \sum_{j, \mu}\left(W_{j, \mu+1}-W_{j, \mu}\right)^{2}},
$$

$P\left(\left\{h_{\mathrm{f}}\right\}, t \mid\left\{h_{0}\right\}, t_{0}\right)$ becomes

$$
P\left(\left\{h_{\mathrm{f}}\right\}, t \mid\left\{h_{0}\right\}, t_{0}\right)=\int \mathcal{D}[h] \exp \left(-\frac{1}{\varepsilon} \boldsymbol{S}[h]\right) .
$$

The Onsager-Machlup functional (or stochastic action) $\boldsymbol{S}[h]$ is defined as the time integral of the stochastic Lagrangian

$$
\boldsymbol{S}[h]=\int_{0}^{t} d s \mathcal{L}[h, \dot{h}]
$$

whose discrete version is

$$
\begin{aligned}
\mathcal{L}[h, \dot{h}]= & \frac{1}{2} \sum_{j}\left(\frac{h_{j, \mu+1}-h_{j, \mu}}{\tau}-\left[\alpha \boldsymbol{L}_{j, \mu+1}+(1-\alpha) \boldsymbol{L}_{j, \mu}\right]\right)^{2} \\
& -2 \nu \alpha N-\alpha \frac{\lambda}{2} \sum_{j}\left[h_{j+1, \mu}-2 h_{j, \mu}+h_{j-1, \mu}\right]
\end{aligned}
$$


The discrete form of the action results to be

$$
\begin{aligned}
\boldsymbol{S}[h]= & \frac{1}{2 \tau} \sum_{j, \mu}\left\{h_{j, \mu+1}-h_{j, \mu}-\tau\left[\alpha \boldsymbol{L}_{j, \mu+1}+(1-\alpha) \boldsymbol{L}_{j, \mu}\right]\right\}^{2} \\
& -2 \nu \alpha N t-\tau \alpha \frac{\lambda}{2} \sum_{j, \mu}\left[h_{j+1, \mu}-2 h_{j, \mu}+h_{j-1, \mu}\right] .
\end{aligned}
$$

As a last convenient discretization, and in the spirit of cellular-automaton calculations often resorted to, we consider the values of $h$ to be integers. The algorithm is then the following: At time $\mu$ a series of trials is made, consisting in increasing by unity the height of a randomly selected site. Out of those trials, we keep the one which gives the least action increase. This process was repeated $\mathcal{O}\left(10^{2}\right)$ times, to obtain meaningful averages.

\section{Analysis of the action: Numerical results}

In order to obtain information about the dynamics, we have carried out an analysis of the action's behavior, as well as the possibility of computing the transition probabilities or the generating functions. To this end, the Lagrangian was split into its components and the relative weight of each contribution was determined. It clearly appears that after an initial transient dominated by the linear EW-like contribution, the nonlinear KPZ contribution becomes dominant.

Figure 1 shows the crossover from the EW regime to the KPZ one. We take as estimator of such a transition the time at which the difference (dotted/black line) between KPZ (solid/red curve) and EW actions (dashdotted/blue line) crosses the EW one (it grossly coincides with the time at which the asymptotes crosses). Other estimator has been considered, namely the time at which the KPZ action "detaches" from the EW one, i.e. the time at which the difference becomes positive. In Fig. 2, we have plotted the dependence of the former estimator on the value of $\lambda$. For comparison, we have also included the trend for $\lambda^{-\phi}$ with $\phi=1.35$ (dotted line). Other preliminary results for $\lambda>7$ seem to indicate a marked change in the value of $\phi$, that maybe could be associated to the entering into a strong coupling region [37]. This estimator has so far only qualitative value, since numerically it agrees neither with the results in [14] (where a value of $\phi \sim 4$ was found) nor with the one in [15] (with $\phi \sim 3$, but corresponding to the $2 \mathrm{D}$ case).

Let us remark again that these results are preliminary because they are strongly affected by the presence of a long-time numerical instability as discussed in the next section. 


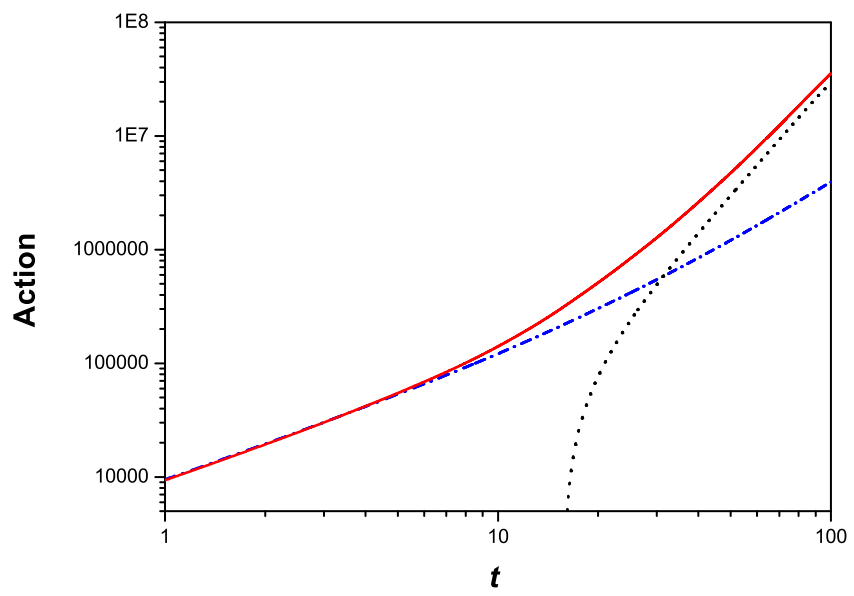

Fig. 1. Tentative numerical analysis of the crossover from the EW regime to the KPZ one. The different actions are: EW action (dash-dotted/blue line), KPZ action (solid/red line), and the difference between both (dotted/black line). Parameter values are $\nu=1, \varepsilon=1, \lambda=1$, lattice size $N_{0}=1028$ sites.

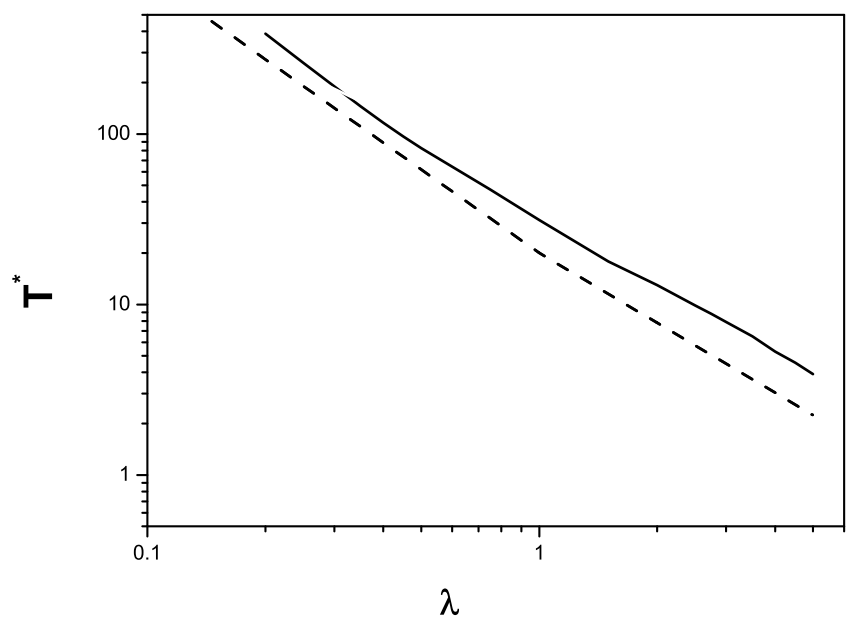

Fig. 2. Crossover time $T^{*}$ vs $\lambda$ (solid/black line). The trend for $T^{*} \sim \lambda^{-\alpha}$ with $\alpha=1.35$ (dashed line) is included for comparison. Other parameters, as indicated in the previous figure.

\section{Conclusions}

A novel path-integral approach is put forward to numerically follow the behavior of the stochastic action of an interface whose growth is described by the KPZ equation. This method, which resorts to a cellular automata-like 
approach and a minimization procedure, effectively acts as an importancesampling method, is intended to predict the crossover time as a function of the coefficient of the nonlinear term, through the comparison of terms in the stochastic action.

Along this work, we have used a lattice size of $N_{0}=1028$ sites. As could be expected, the results depend on the system size. A detailed analysis of such a size dependence, particularly on the crossover time (for the different estimators), as well as the effect of dimensionality will be the subject of future work.

An extremely important point is that the action, for long times, should grow linearly in time

$$
\tilde{S}[h] \sim \int\left\langle\xi\left(x^{\prime}, t^{\prime}\right) \xi\left(x^{\prime \prime}, t^{\prime \prime}\right)\right\rangle d x^{\prime} d x^{\prime \prime} d t^{\prime} d t^{\prime \prime} \sim t .
$$

Our results show no trace of this behavior, and instead what we see is a much faster growth of the action. This reveals that the present algorithm shows some instability that dominates the long time evolution. We leave for future work the analysis of the origin of this instability and its possible remedies. For the sake of completeness, let us mention that different instabilities for this type of system were observed in other works [7, 9, 10].

Financial support from MINECO (Spain) through Projects FIS201018023 (H.S.W. and C.E.) and RYC-2011-09025 (C.E.) is acknowledged. R.R.D. acknowledges financial support from UNMdP (Argentina) through Project EXA544/11 and thanks warmly MECD (Spain) for its support through SAB2011-0079.

\section{REFERENCES}

[1] K.G. Wilson, Scientific American, August, 1979.

[2] M. Kardar, G. Parisi, Y.-C. Zhang, Phys. Rev. Lett. 56, 889 (1986).

[3] T. Halpin-Healy, Y.-C. Zhang, Phys. Rep. 254, 215 (1995).

[4] A.L. Barabasi, H.E. Stanley, Fractal Concepts in Surface Growth, Cambridge U. Press, Cambridge, UK, 1995.

[5] V. Gurarie, A. Migdal, Phys. Rev. E54, 4908 (1996).

[6] M. Kardar, Nucl. Phys. B290, 582 (1987).

[7] M. Beccaria, G. Curci, Phys. Rev. E50, 4560 (1994).

[8] K. Moser, D.E. Wolf, J. Phys. A: Math. Gen. 27, 4049 (1994).

[9] M. Scalerandi, P.P. Delsanto, S. Biancotto, Comput. Phys. Commun. 97, 195 (1996). 
[10] T.J. Newman, A.J. Bray, J. Phys. A: Math. Gen. 29, 7917 (1996).

[11] C. Appert, Comput. Phys. Commun. 121, 363 (1999).

[12] E. Marinari, A. Pagnani, G. Parisi, J. Phys. A: Math. Gen. 33, 8181 (2000).

[13] L. Giada, A. Giacometti, M. Rossi, Phys. Rev. E65, 036134 (2002).

[14] B.M. Forrest, R. Toral, J. Stat. Phys. 70, 703 (1993).

[15] H. Guo, B. Grossmann, M. Grant, Phys. Rev. A41, 7082 (1990).

[16] T. Sasamoto, H. Spohn, Phys. Rev. Lett. 104, 230602 (2010).

[17] G. Amir, I. Corwin, J. Quastel, Commun. Pure Appl. Math. 64, 466 (2011).

[18] P. Calabrese, P. Le Doussal, Phys. Rev. Lett. 106, 250603 (2011).

[19] C. Escudero, Phys. Rev. E84, 031131 (2011).

[20] C. Escudero, Chaos Solitons Fractals 45, 109 (2012).

[21] W.D. McComb, Phys. Rev. E71, 037301 (2005).

[22] A. Berera, D. Hochberg, Phys. Rev. Lett. 99, 254501 (2007).

[23] A. Berera, D. Hochberg, Nucl. Phys. B814, 522 (2009).

[24] M. Nicoli, R. Cuerno, M. Castro, Phys. Rev. Lett. 102, 256102 (2009).

[25] H.S. Wio et al., Europhys. Lett. 89, 40008 (2010).

[26] H.S. Wio et al., Phys. Rev. E81, 066706 (2010).

[27] H.S. Wio et al., Philos. Trans. R. Soc. Lond. A369, 396 (2011).

[28] H.S. Wio, Int. J. Bifurcation Chaos 18, 2813 (2009).

[29] C. Escudero et al., KPZ Equation as a Gradient Flow:

Nonequilibrium-potential Expansion and Renormalization-group Treatment of Fluctuations, submitted for publication.

[30] T. Sun, H. Guo, M. Grant, Phys. Rev. A40, 066706 (1989).

[31] J. Villain, J. Phys. I (France) 1, 19 (1991).

[32] Z.-W. Lai, S. Das Sarma, Phys. Rev. Lett. 66, 2348 (1991).

[33] C. Escudero, Phys. Rev. Lett. 101, 196102 (2008).

[34] C. Escudero, E. Korutcheva, J. Phys. A: Math. Theor. 45, 125005 (2012).

[35] C. Escudero, I. Peral, J. Differ. Equ. 254, 2515 (2013).

[36] C. Escudero, R. Hakl, I. Peral, P.J. Torres, Eur. J. Appl. Math. 24, 437 (2013).

[37] H.C. Fogedby, W. Ren, Phys. Rev. E80, 041116 (2009).

[38] H.S. Wio, Application of Path Integration to Stochastic Processes: An Introduction, World Scientific, Singapore 2013.

[39] F. Langouche, D. Roekaerts, E. Tirapegui, Functional Integration and Semiclassical Expansions, D. Reidel Pub. Co., Dordrecht 1982. 